\title{
Increased fatty acid synthase expression in prostate biopsy cores predicts higher Gleason score in radical prostatectomy specimen
}

Shinsuke Hamada ${ }^{1 *}$, Akio Horiguchi ${ }^{1}$, Kenji Kuroda ${ }^{1}$, Keiichi Ito ${ }^{1}$, Tomohiko Asano ${ }^{1}$, Kosuke Miyai ${ }^{1,2}$ and Keiichi Iwaya ${ }^{1,2}$

\begin{abstract}
Background: Fatty acid synthase (FAS) is highly expressed in various types of cancer, and elevated expression of FAS has been suggested to be a predictor of tumor aggressiveness and poor prognosis. We examined whether FAS expression in prostate biopsy cores could predict the pathological characteristics of radical prostatectomy (RP) specimens.

Methods: Paraffin-embedded prostate biopsy cores, obtained from 102 patients who subsequently underwent RP, were immunostained with polyclonal anti-FAS antibody. The staining intensity was categorized into non-staining, weak, moderate, and strong. Tumors with moderate or strong immunostaining were considered to show high FAS expression, and other tumors were considered to show low FAS expression. The relation between the FAS expression status in biopsy cores and pathological parameters in RP specimens was analyzed.

Results: The FAS expression in the biopsy cores of 64 of the 102 tumors (63\%) was high, whereas it was low in the biopsy cores of the other 38 tumors (37\%). High FAS expression was significantly associated with Gleason Score $(G S) \geq 7$ in RP specimens ( $p<0.0001$ ). In multivariable logistic regression analyses, $G S \geq 7$ in biopsy cores $(p<0.0001)$, higher preoperative PSA $(p=0.0194)$, and high FAS expression $(p=0.0004)$ were independent predictors of $G S \geq 7$ in the RP specimen.

Conclusions: Increased FAS expression in prostate biopsy cores could be a novel parameter for predicting higher GS in RP specimens. The treatment strategy for patients with high FAS expression in prostate biopsy cores should be carefully determined.
\end{abstract}

Keywords: Fatty acid synthase, Prostate cancer, Biopsy core, Gleason score

\section{Background}

Fatty acids are essential constitutions of all biological membrane lipids, and are very important substrates for energy metabolism [1]. The biosynthesis of endogenously synthesized fatty acids is catalyzed by multifunctional homodimeric fatty acid synthase (FAS) [1,2]. FAS is a biosynthetic enzyme in lipogenesis and the production of long-chain fatty acids from acetyl-coenzyme A (Co A), malonyl-CoA, and nicotinamide adenine dinucleotide phosphate (NADPH) [1-3]. Under normal conditions,

\footnotetext{
* Correspondence: shinsukehamaji@yahoo.co.jp

'Department of Urology, National Defense Medical College, 3-2 Namiki,

Tokorozawa-City, Saitama 359-8513, Japan

Full list of author information is available at the end of the article
}

FAS converts excess carbohydrates into fatty acids that are then esterified to storage triacylglycerols and provide energy through $\beta$-oxidation $[1,2]$. Except for liver and adipose cells, normal cells in humans have low or undetectable levels of expression and activity of FAS, which is closely regulated by diet, hormones, and growth factors [1,2].

On the other hand, FAS is highly expressed in various types of cancer, and elevated expression of FAS has been linked to tumor aggressiveness and poor prognosis and reduced disease-free survival [4-6]. FAS was first identified as an oncogenic antigen 519 (OA 519) in patients with breast cancer and a markedly poorer prognosis [7]. FAS expression is an indicator of poor prognosis in 
breast and prostate cancers and is elevated in the blood of cancer patients [3].

However, to the best of our knowledge, the predictive value of FAS expressed in prostate biopsy cores has yet to be clarified as to the clinicopathological parameters of prostate cancer. On the basis of the studies mentioned above, we hypothesized that the expression status of FAS in prostate biopsies might provide valuable information for clinicians. In this study, we performed an immunohistochemical analysis of FAS expression in prostate biopsy cores and a statistical analysis on the relationship between the expression status of FAS prostate biopsy cores and clinicopathological parameters.

\section{Methods}

\section{Patients}

This study included 102 patients (median age 67, range 51-75) who had underwent ultrasonically guided transrectal and/or transperinieal prostate biopsies and radical prostatectomies (RP) for clinically localized prostate cancers at the National Defense Medical College from May 1998 to April 2007. Patient characteristics are listed on Table 1. Clinical $\mathrm{T}$ stage was assessed by digital rectal examination and/or transrectal ultrasound in accordance with the 2009 TNM classification. The Gleason grading system for biopsies and surgical specimens was in accordance with the 2005 International Society of Urological Pathology (ISUP 2005) [8]. The positive core rate was defined as the number of positive biopsy cores divided by total number of biopsy cores. The levels of serum PSA were measured on the day before the operation. Patients who received hormonal therapy or radiation therapy prior

\section{Table 1 Patient characteristics}

\begin{tabular}{ll}
\hline Parameters & No. (\%) \\
\hline Over all & 102 \\
Median age (range) & $67(51-75)$ \\
Clinical T stage & \\
CT1c & $71(69.6)$ \\
cT2a & $17(16.7)$ \\
CT2b & $3(2.9)$ \\
CT2c & $0(0)$ \\
CT3 & $11(10.8)$ \\
PSA (ng/ml) & \\
0-4 & $2(2.0)$ \\
$4-10$ & $58(56.9)$ \\
$10-20$ & $25(24.5)$ \\
$20-$ & $17(16.6)$ \\
median (range) & $8.172(3.3-61.9)$ \\
Total biopsy cores (median) & $6-24(8)$ \\
Positive core rate (\%) (median) & $5.56-100(25)$ \\
\hline
\end{tabular}

to RP were excluded from the analysis. The follow-up period calculated from the date of the operation to the date of the last recorded follow-up ranged 1.0 to 149.5 months (median: 73 months). PSA failure was considered to have occurred if PSA value was over $0.2 \mathrm{ng} / \mathrm{ml}$ after RP. We obtained informed consent for participation in the study from participants. This study was approved by the National Defense Medical College ethical committee.

\section{Immunohistochemical analyses of FAS expression in prostate biopsy cores}

Formalin-fixed, paraffin-embedded biopsy cores, $4 \mu \mathrm{m}$ thick, were deparaffinized in xylene and rehydrated through graded ethanols. Endogenous peroxidase activity was quenched with DAKO Peroxidase Blocking Reagent (Dako Corporation) for 10 minutes. The slides were incubated in $10 \%$ normal goat serum in PBS for 60 minutes at room temperature. After the slides were incubated 60 minutes in a 1:50 dilution of rabbit polyclonal anti-FAS antibody (Immuno-Biological Laboratories Co., Fujioka, Japan) in PBS, the slides were stained by using a Simple Stain Max PO (Nichirei Corporation, Tokyo, Japan) according to the manufacturer's instructions. Reaction products were visualized by immersing the slides in DAB for 2 minutes. The immunohistochemistry was evaluated independently by 2 observers (K.M. and K.I.) blinded from any clinical data. Whole biopsy slides were evaluated in all cases examined. While comparing with the slides of hematoxylin-eosin stain, only prostatic cancer cells were taken into account for the evaluation of FAS protein expression. Cytoplasmic intensities of FAS immunoreactivity were classified semiquantitatively into the four categories: non-staining (negative), weak (score 1+), moderate (score $2+$ ), and strong (score $3+$ ) $[9,10]$. When there were heterogeneous intensities of FAS immunoreactivity within examined cases, strongest intensity was adapted as the score of FAS expression in each case. The staining intensity in normal prostate gland was used as an internal control. Any discrepancies between the assessments of the 2 observers were resolved by conferring over a multiviewer microscope. Tumors with $3+$ or $2+$ immunostaining were considered high, and other tumors were considered low. The Gleason score (GS) in the biopsy core was determined on the same sections.

\section{Statistical analysis}

All statistical analyses were performed by using the JMP 9.0 soft-ware system for Windows (SAS Institute Inc. Cary, NC, USA). A chi-square test was used to assess the association between preoperative and pathological specimen parameters relative to the level of FAS expression. Logistic regression analyses were used for multivariate 


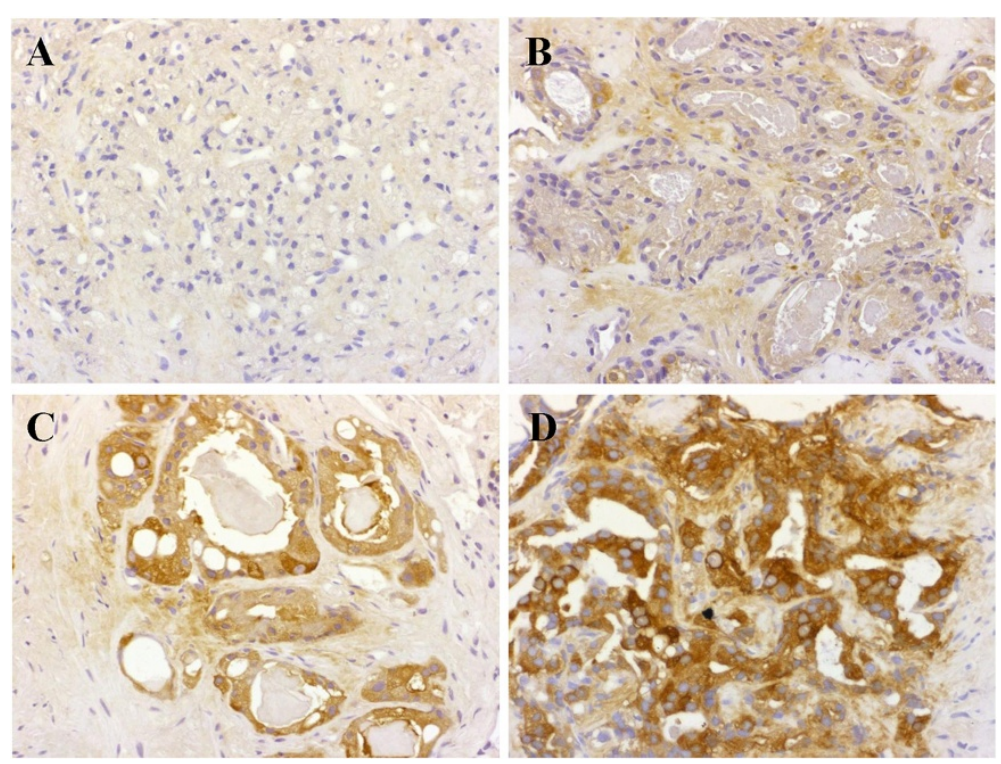

Figure 1 Representative immunohistochemistry for fatty acid synthase (FAS) in prostatic adenocarcinoma. A, Case of prostatic adenocarcinoma showing no immunoreactivity for FAS (score 0). B-D, Cases of prostatic adenocarcinoma showing weak (B), moderate (C), and strong (D) cytoplasmic immunoreactivity for FAS, and scored as 1+,2+, and 3+, respectively. Immunoperoxidase stain, original magnification x200

analyses to predict RP specimen GS $\geq 7$. Receiver operating characteristic (ROC) curves were plotted with the sensitivity (true-positive fraction) on the $y$-axis versus 1 minus the specificity (false-positive fraction) on the $x$-axis. The PSA failure rate was calculated and compared by log-rank testing. $\mathrm{P}$ values less than 0.05 were considered to indicate statistical significance.

\section{Results}

Relations between FAS expression in biopsy cores and preoperative parameters

We first evaluated the expression level of FAS in prostate biopsy cores. FAS expression was high in the biopsy cores of 64 of the 102 tumors (63\%) and low in the other 38 tumors (37\%): 4 (4\%) showing negative immunostaining, 34 (33\%) showing 1+ immunostaining (weak staining), 44 (43\%) showing $2+$ immunostaining (moderate staining), 20 (20\%) showing 3+ immunostaining (strong staining) (Figure 1). Table 2 lists the $\mathrm{p}$ values for the associations between FAS status and preoperative parameters. High FAS expression was significantly associated with clinical T stage (cT2-cT3) ( $\mathrm{p}=0.0381), \mathrm{GS} \geq 7$ in the biopsy core $(\mathrm{p}=0.0044)$ and positive core rate $>40 \%(\mathrm{p}=0.0038)$. Preoperative parameters including PSA and D'Amico risk classification [11] were not associated with FAS expression status (Table 2).

\section{Relations between FAS expression and pathological parameters of RP specimens}

Next, we analyzed the association between FAS expression in the biopsy cores and pathological features of RP specimens. High FAS expression was significantly associated with GS $\geq 7$ in the RP specimens $(\mathrm{p}<0.0001$, Table 3). However, high FAS expression was not significantly associated with any other pathological parameters, including status of extraprostatic extention, lymphatic invasion, microvessel invasion, or perineural invasion. Although high FAS expression in biopsy cores was significantly associated with positive core rate $>40 \%$, it was not associated with maximum tumor diameter in RP specimens.

Table 2 FAS expression and preoperative parameters

\begin{tabular}{|c|c|c|c|c|}
\hline \multirow[b]{2}{*}{ Parameters } & \multirow[b]{2}{*}{ Patients } & \multicolumn{2}{|c|}{ FAS expression } & \multirow[b]{2}{*}{$P$ value } \\
\hline & & Low & High & \\
\hline \multicolumn{5}{|l|}{ Clinical T stage } \\
\hline cT1c & 71 & 31 & 40 & 0.0381 \\
\hline сT2-сT3 & 31 & 7 & 24 & \\
\hline \multicolumn{5}{|l|}{ PSA (ng/ml) } \\
\hline$\leq 10$ & 60 & 24 & 36 & 0.4919 \\
\hline $10<$ & 42 & 14 & 28 & \\
\hline \multicolumn{5}{|l|}{ Needle biopsy Gleason score } \\
\hline$\leq 6$ & 31 & 18 & 13 & 0.0044 \\
\hline $7 \leq$ & 71 & 20 & 51 & \\
\hline \multicolumn{5}{|l|}{ Positive core rate (\%) } \\
\hline$\leq 40$ & 72 & 33 & 39 & 0.0038 \\
\hline $40<$ & 30 & 5 & 25 & \\
\hline \multicolumn{5}{|l|}{ D’Amico risk classification } \\
\hline Low and Intermediate risk & 67 & 28 & 39 & 0.1853 \\
\hline High risk & 35 & 10 & 25 & \\
\hline
\end{tabular}


Table 3 FAS expression and pathological parameters

\begin{tabular}{|c|c|c|c|c|}
\hline \multirow[b]{2}{*}{ Parameters } & \multirow[b]{2}{*}{ Patients } & \multicolumn{2}{|c|}{ FAS expression } & \multirow[b]{2}{*}{$P$ value } \\
\hline & & Low & High & \\
\hline \multicolumn{5}{|c|}{ Gleason score } \\
\hline$\leq 6$ & 23 & 18 & 5 & $<0.0001$ \\
\hline $7 \leq$ & 79 & 20 & 59 & \\
\hline \multicolumn{5}{|c|}{ Extra-prostatic extension } \\
\hline Positive & 45 & 14 & 31 & 0.3002 \\
\hline Negative & 56 & 23 & 33 & \\
\hline \multicolumn{5}{|c|}{ Lymphatic invasion } \\
\hline Positive & 51 & 18 & 33 & 0.6821 \\
\hline Negative & 51 & 20 & 31 & \\
\hline \multicolumn{5}{|c|}{ Microvascular invasion } \\
\hline Positive & 40 & 13 & 27 & 0.4232 \\
\hline Negative & 62 & 25 & 37 & \\
\hline \multicolumn{5}{|c|}{ Perineural invasion } \\
\hline Positive & 71 & 23 & 48 & 0.0978 \\
\hline Negative & 30 & 15 & 15 & \\
\hline \multicolumn{5}{|c|}{ Maximum tumor size } \\
\hline$\leq 10 \mathrm{~mm}$ & 13 & 6 & 7 & 0.4823 \\
\hline $10 \mathrm{~mm}<$ & 89 & 32 & 57 & \\
\hline
\end{tabular}

High FAS expression in biopsy cores is an independent predictor of GS $\geq 7$ in RP specimens

The ability to predict GS in RP specimens using preoperative parameters is quite important for the selection of therapies for localized prostate carcinoma [12,13]. On the basis of our results, we examined whether the FAS expression in biopsy cores could be a novel preoperative predictor of higher GS in the RP specimens. In the univariate analysis, preoperative parameters including clinical $\mathrm{T}$ stage, GS $\geq 7$ in biopsy cores, PSA, positive core rate, and high FAS expression in biopsy cores were significantly associated with $\mathrm{GS} \geq 7$ in RP specimens. In the multivariable logistic regression analyses, biopsy GS $\geq 7$ ( $p<0.0001)$, increasing preoperative serum PSA level $(p=0.0202)$, and high FAS expression $(\mathrm{p}=0.0012)$ were independent predictors of GS $\geq 7$ in RP specimens (Table 4).

\section{ROC analyses}

The area under the ROC curve (AUC) was calculated (Figure 2) as a way to estimate the predictive value of the preoperative parameters for RP specimens with $\mathrm{GS} \geq 7$. AUC was 0.6277 for PSA, 0.9062 for needle biopsy GS, 0.6263 for clinical T stage, 0.8079 for D'Amico risk classification, and 0.7933 for FAS expression. In particular, the AUC of the combination of FAS and GS $\geq 7$ in the biopsy cores reached 0.9623 .

\section{FAS expression and the period to PSA failure}

The Kaplan-Meier survival curves for PSA fairure rate is shown in Figure 3, where they are stratified by FAS expression. Although patients with high FAS expression tended to have high PSA failure rates compared with those with low FAS expression, it did not reach statistically significant $(\mathrm{p}=0.1028)$.

\section{Discussion}

Fatty acids are synthesized to provide lipids for energy production via $\beta$-oxidation and lipid modification of proteins and membrane formation in cancer cells [1-3]. FAS is highly expressed in many cancers, including thyroid, oral, tongue, esophageal, lung, breast, endometrial, ovarian, colorectal, bladder, hepatocellular, pancreatic and gastric carcinomas, as well as sarcoma, Paget's disease of the vulva, gastrointestinal stromal tumor, and multiple myeloma [1-3,14-16]. Regarding urological malignancies, increased expression and its association with tumor aggressiveness and poor cancer-specific survival are evident in patients with prostate and kidney cancer [9,17-19].

The relationship between FAS and prostate cancer has been widely investigated. FAS was over-expressed in high grade prostatic epithelial neoplasia (PIN), and FAS expression was found to be possibly one of the earliest events in the development of prostate cancer [9]. FAS expression was also a strong independent predictor of pathologic stage [17]. While FAS expression in benign tissue was reduced after androgen ablation, it persisted in human prostate carcinoma after androgen ablation and was high in $82 \%$ of lethal tumors examined by autopsy [18]. Increased FAS expression appeared to increase the

Table 4 Univariate and multivariate analyses to predict specimen Gleason score $\geq 7$

\begin{tabular}{|c|c|c|c|c|c|c|}
\hline & \multicolumn{3}{|c|}{ Univariate } & \multicolumn{3}{|c|}{ Multivariate } \\
\hline & $P$ value & Odds ratio & $95 \% \mathrm{Cl}$ & $P$ value & Odds ratio & $95 \% \mathrm{Cl}$ \\
\hline FAS expression in biopsy cores (Low vs. High) & $<0.0001$ & 10.62 & $3.71-35.65$ & 0.0012 & 34.15 & $3.42-1145.4$ \\
\hline Clinical T stage (cT1 vs. cT2 $\leq$ ) & 0.0293 & 3.66 & $1.13-16.49$ & 0.4890 & 2.29 & $0.23-32.36$ \\
\hline Needle biopsy Gleason score ( $\leq 6$ vs. $7 \leq$ ) & $<0.0001$ & 72.45 & $17.84-499.7$ & $<0.0001$ & 228.17 & $25.37-8358.31$ \\
\hline PSA (ng/ml) & 0.0174 & 1.09 & $1.01-1.19$ & 0.0202 & 1.22 & $1.03-1.57$ \\
\hline Positive core rate (\%) ( $\leq 40 \%$ vs. $40 \%<$ ) & 0.007 & 5.76 & $1.53-37.7$ & 0.9106 & 1.14 & $0.10-10.95$ \\
\hline Total biopsy cores ( $\leq 8$ vs. $8<$ ) & 0.4131 & 1.48 & $0.58-3.84$ & 0.9423 & 1.08 & $0.14-8.42$ \\
\hline
\end{tabular}



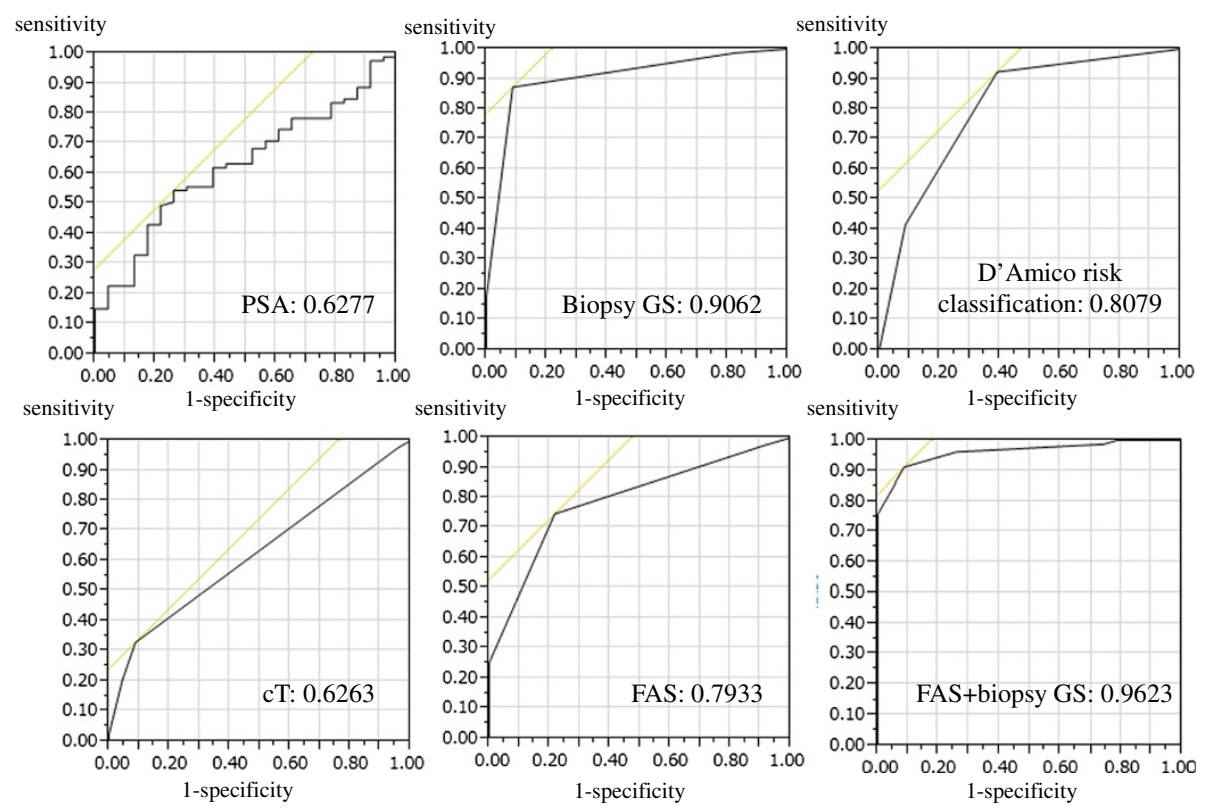

Figure 2 Receiver operating characteristic (ROC) curves and areas under the ROC curves (AUC) of PSA, biopsy Gleason score, D'Amico risk classification, clinical T stage, FAS expression, and needle biopsy Gleason score added to FAS expression for predicting RP specimen Gleason score $\geq 7$.

risk of death from prostate cancer 4.4-fold [6]. FAS may be used as a prostate cancer marker, and anti-neoplastic therapy based on FAS inhibitions may be an option for chemo prevention or curative treatment of nearly all prostate cancers [9]. These observations suggest that FAS expression status could be an important parameter of tumor aggressiveness and in prostate cancer. Although the mechanism of cell malignant transformation or cell proliferation induced by FAS over expression in malignant tumor is not fully understood, several mechanisms including increasing expression of epidermal growth factor receptor and activation of PI3K/AKT and ERK1/2 signaling pathways, and the modulation and nuclear

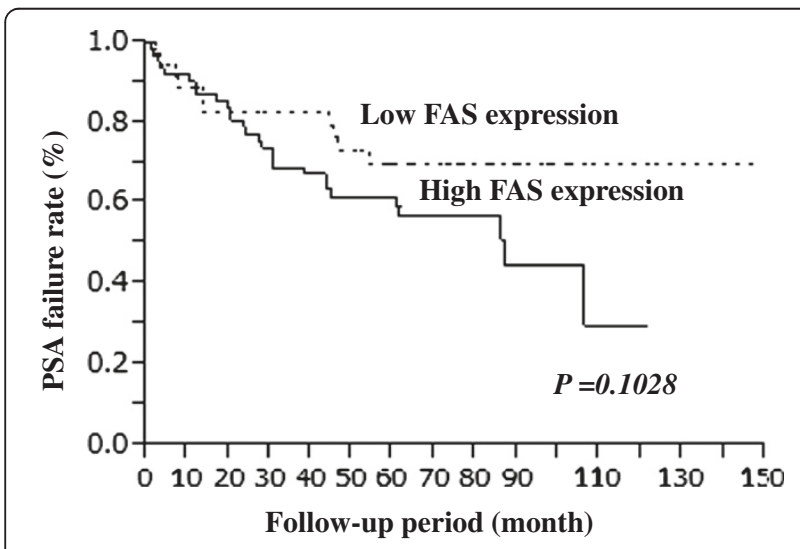

Figure 3 Kaplan-Meier curves for PSA failure rates after RP in patients with high and low FAS expression. maturation of the transcription factor sterol regulatory element-binding protein 1c have been reported [1-3].

Most of the studies on FAS expression in prostate cancer examined RP specimens, and a few studies focused on FAS expression in biopsy cores $[4,6,9,17,18]$. Bandyopadhyay et al. and Swinnen et al. reported that FAS expression levels in prostate cancer specimens including needle biopsies were correlated with the degree of Gleason grade $[6,9]$. In our study's results, high FAS expression in biopsy cores was significantly associated with biopsy cores GS $\geq 7$, which is consistent with previous observations $[6,9]$. We therefore speculated that the expression status of FAS in the biopsy core could be a novel diagnostic tool for evaluating tumor aggressiveness in prostate cancer. The decision of which treatment to pursue in clinically localized prostate cancer depends on the aggressiveness of the tumor. GS in biopsy cores is one of the most valuable parameters of tumor aggressiveness. The ability to assign a precise GS in the prostate is quite important for selecting appropriate treatments for prostate cancer. However, the GS values in about $40-50 \%$ of RP specimens do not correspond with those calculated from needle biopsies [12,13,20]. Various parameters including patient age, PSA, maximum percentage cancer/biopsy cores, the number of biopsy cores and surgical specimen weight have been shown to be useful for precisely predicting GS $[12,20]$ in RP specimens, but none has proven to be definitive. In our study, high FAS expression of biopsy cores was an independent predictor of RP specimens $G S \geq 7$ in addition to GS of biopsy cores. In 
particular, the AUC of the combination of FAS and GS $\geq 7$ in biopsy cores reached 0.9623 .

Our studies have some limitations. Although we analyzed the relationship between FAS expression and the period to PSA failure, there was no significant association between the parameters. We consider that the underlying reasons include insufficient numbers of patients and RPs performed by variety of surgeons with different skills. We could not analyze the relationship between nutritional status and FAS expression levels. In recent study, it has been reported that overweight prostate cancer patients tend to have germline single nucleotide polymorphisms (SNPs) in the FAS gene and FAS tumor expression, suggesting FAS might play the key role in poor outcome in obese prostate cancer patients [21]. Despite such limitations, our results provide novel information about biopsy cores for precisely predicting GS.

\section{Conclusions}

Increased FAS expression in prostate biopsy cores could be a novel parameter for predicting higher GS in RP specimens. The treatment strategy for patients with high FAS expression in prostate biopsy cores should be carefully determined.

\section{Abbreviations}

FAS: Fatty acid synthase; RP: Radical prostatectomy; GS: Gleason score; ROC: Receiver operating characteristic; AUC: The area under the ROC curve.

\section{Competing interests}

The authors declare that they have no competing interests.

\section{Authors' contributions}

$\mathrm{SH}$ and $\mathrm{AH}$ were responsible for drafting the manuscript. $\mathrm{KM}$ and $\mathrm{KI}$ contributed to immunohistochemistry. KK, KI and TA contributed to analysis and interpretation of data. All authors read and approved the final manuscript.

\section{Author details}

${ }^{1}$ Department of Urology, National Defense Medical College, 3-2 Namiki, Tokorozawa-City, Saitama 359-8513, Japan. ²Department of Basic Pathology, National Defense Medical College, Tokorozawa-City, Saitama, Japan.

Received: 22 July 2013 Accepted: 9 January 2014

Published: 14 January 2014

\section{References}

1. Menendez JA, Lupu R: Fatty acid synthase and the lipogenic phenotype in cancer pathogenesis. Nat Rev Cancer 2007, 7:763-777.

2. Flavin R, Peluso S, Nguyen PL, Loda M: Fatty acid synthase as a potential therapeutic target in cancer. Future Oncol 2010, 6:551-562.

3. Kuhajda FP: Fatty acid synthase and cancer: new application of an old pathway. Cancer Res 2006, 66:5977-5980.

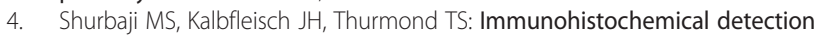
of a fatty acid synthase (OA-519) as a predictor of progression of prostate cancer. Hum Pathol 1996, 27:917-921.

5. Takahiro T, Shinichi K, Toshimitsu S: Expression of fatty acid synthase as a prognostic indicator in soft tissue sarcomas. Clin Cancer Res 2003, 9:2204-2212.

6. Bandyopadhyay S, Pai SK, Watabe M, Gross SC, Hirota S, Hosobe S, Tsukada T, Miura K, Saito K, Markwell SJ, et al: FAS expression inversely correlates with PTEN level in prostate cancer and a PI 3-kinase inhibitor synergizes with FAS siRNA to induce apoptosis. Oncogene 2005, 24:5389-5395.
7. Kuhajda FP, Jenner K, Wood FD, Hennigar RA, Jacobs LB, Dick JD, Pasternack GR: Fatty acid synthesis: a potential selective target for antineoplastic therapy. Proc Natl Acad Sci U S A 1994, 91:6379-6383.

8. Epstein Jl, Allsbrook WC Jr, Amin MB, Egevad LL: The 2005 International Society of Urological Pathology (ISUP) consensus conference on Gleason grading of prostatic carcinoma. Am J Surg Pathol 2005, 29:1228-1242.

9. Swinnen JV, Roskams T, Joniau S, van Poppel H, Oyen R, Baert L, Heyns W, Verhoeven G: Overexpression of fatty acid synthase is an early and common event in the development of prostate cancer. Int J Cancer 2002, 98:19-22.

10. Migita T, Ruiz S, Fornari A, Fiorentino M, Priolo C, Zadra G, Inazuka F, Grisanzio C, Palescandolo E, Shin E, et al: Fatty acid synthase: a metabolic enzyme and candidate oncogene in prostate cancer. J Natl Cancer Inst 2009, 101:519-532

11. D'Amico AV, Whittington R, Malkowicz SB, Schultz D, Blank K, Broderick GA, Tomaszewski JE, Renshaw AA, Kaplan I, Beard CJ, Wein A: Biochemical outcome after radical prostatectomy, external beam radiation therapy, or interstitial radiation therapy for clinically localized prostate cancer. JAMA 1998, 280:969-974.

12. Epstein Jl, Feng Z, Trock BJ, Pierorazio PM: Upgrading and downgrading of prostate cancer from biopsy to radical prostatectomy: incidence and predictive factors using the modified Gleason grading system and factoring in tertiary grades. Eur Urol 2012, 61:1019-1024.

13. Iremashvili V, Manoharan M, Pelaez L, Rosenberg DL, Soloway MS: Clinically significant Gleason sum upgrade: external validation and head-to-head comparison of the existing nomograms. Cancer 2012, 118:378-385.

14. Kuhajda FP, Pizer ES, Li JN, Mani NS, Frehywot GL, Townsend CA: Synthesis and antitumor activity of an inhibitor of fatty acid synthase. Proc Natl Acad Sci U S A 2000, 97:3450-3454.

15. Menendez JA, Lupu R: Oncogenic properties of the endogenous fatty acid metabolism: molecular pathology of fatty acid synthase in cancer cells. Curr Opin Clin Nutr Metab Care 2006, 9:346-357.

16. Swinnen JV, Brusselmans K, Verhoeven G: Increased lipogenesis in cancer cells: new players, novel targets. Curr Opin Clin Nutr Metab Care 2006 9:358-365.

17. Epstein Jl, Carmichael M, Partin AW: OA-519 (fatty acid synthase) as an independent predictor of pathologic state in adenocarcinoma of the prostate. Urology 1995, 45:81-86.

18. Pizer ES, Pflug BR, Bova GS, Han WF, Udan MS, Nelson JB: Increased fatty acid synthase as a therapeutic target in androgen-independent prostate cancer progression. Prostate 2001, 47:102-110.

19. Horiguchi A, Asano T, Ito K, Sumitomo M, Hayakawa M: Fatty acid synthase over expression is an indicator of tumor aggressiveness and poor prognosis in renal cell carcinoma. J Urol 2008, 180:1137-1140.

20. Thomas C, Pfirrmann K, Pieles F, Bogumil A, Gillitzer R, Wiesner C, Thuroff JW, Melchior SW: Predictors for clinically relevant Gleason score upgrade in patients undergoing radical prostatectomy. BJU Int 2012, 109:214-219.

21. Nguyen PL, Ma J, Chavarro JE, Freedman ML, Lis R, Fedele G, Fiore C, Qiu W, Fiorentino M, Finn S, et al: Fatty acid synthase polymorphisms, tumor expression, body mass index, prostate cancer risk, and survival. J Clin Oncol 2010, 28:3958-3964.

doi:10.1186/1472-6890-14-3

Cite this article as: Hamada et al:: Increased fatty acid synthase expression in prostate biopsy cores predicts higher Gleason score in radical prostatectomy specimen. BMC Clinical Pathology 2014 14:3. 